\title{
On General Iterative Methods for the Solutions of a Class of Nonlinear Operator Equations*
}

\author{
By M. Z. Nashed
}

1. Introduction. General iterative methods based on the notions of the relative error functional and the projection are introduced for the solutions of a class of nonlinear operator equations in Hilbert space. It is shown that general iterative procedures based on the variational theory of operators (which include the classical steepest descent method and other gradient methods) are first-order approximations in the sense of the Fréchet differential to the methods introduced in this paper.

The former procedures are closely related to the recent works of M. M. Valnberg [18], [19], Ju. Lumiste [11], the author [13] and others (see [13], [18]). These references contain various proofs of convergence of the method of steepest descent for certain classes of nonlinear operator equations. Proofs of convergence of some of the other procedures described here will appear in a separate paper.

When specialized to linear operators, the results may provide a broader unifying interpretation for a number of iterative methods for the solutions of linear operator equations developed by Altman [1], Gastinel [2], Hayes [4], Hestenes [5], Kantorovich [7], Krasnosel'skir and Krel̆n [8], Lanczos [10], Petryshyn [14] and others. It is worth noting that the formulation of various problems in numerical analysis in the framework of functional analysis unifies different problems and methods and sometimes leads to illuminating insight as reflected in the contributions of $M$. Altman, L. Collatz, L. V. Kantorovich, J. Schröder, and others.

This paper also extends to nonlinear operators certain properties of known algorithms for iterative solutions of linear operator equations.

The author is greatly indebted to Professor R. C. F. Bartels for many stimulating discussions on iterative methods, and to the referee for helpful recommendations.

2. Preliminaries. We begin by recalling a few definitions and results. Let $E$ and $Y$ be two normed linear spaces over the field of real numbers and let $X$ be an open subset of $E$. Let $F$ be a mapping of $X$ into $Y$. Let $x_{0}$ be a point of $X$ and let $h$ be an arbitrary element in $E$.

Definition 1. If for every element $h \in E$, and $t$ real,

$$
\left.\frac{d}{d t} F\left(x_{0}+t h\right)\right|_{t=0}
$$

exists, it is called the Gâteaux variation (or the weak differential) of $F$ at $x_{0}$ with increment $h$, and is denoted by $\delta F\left(x_{0} ; h\right)$.

The weak differential is homogeneous in $h$ of degree one.

Received January 6, 1964.

* An abstract was presented to the American Mathematical Society on April 20, 1964 (611-32). 
Definition 2. If $\delta F\left(x_{0} ; h\right)$ is linear and bounded in $h$, it is called the Gâteaux differential of $F$ at $x_{0}$ with increment $h$, and is denoted by $D F\left(x_{0} ; h\right)$.

Let the Gâteaux variation of the operator $F(x)$ exist in some neighborhood $N\left(x_{0}\right)$ of $x_{0}$ and let $\delta F(x ; h)$ be continuous in $x$ at $x_{0}$. Furthermore, assume that $\delta F(x ; \cdot)$ is a continuous operator in $h$ at the origin $h=\theta$. Then $\delta F(x ; h)$ is a Gâteaux differential [17].

The following characterization of the Gâteaux differential follows directly from Definitions 1 and 2. A necessary and sufficient condition for $F(x)$ to be Gâteaux differentiable at $x=x_{0}$ is the existence of a mapping $L\left(x_{0} ; h\right)$ which is linear and bounded in $h$ such that for any $h$ in the $x_{0}$-translate of $X$, i.e., $h \in X-x_{0}$, the following representation holds

$$
F\left(x_{0}+h\right)-F\left(x_{0}\right)=L\left(x_{0} ; h\right)+R\left(x_{0} ; h\right),
$$

where $R\left(x_{0} ; h\right)$ has the property that

$$
\lim _{\tau \rightarrow 0} \frac{\left\|R\left(x_{0} ; \tau h\right)\right\|}{\tau}=0 .
$$

If such a representation exists, it is unique and $L\left(x_{0} ; h\right)=D\left(x_{0} ; h\right)$.

Definition 3. The mapping $F: X \rightarrow Y$ is said to be Fréchet differentiable at the point $x_{0}$ if the representation $(2.1)$ holds, where $L(x ; h)$ is bounded and linear in $h$, and the following is satisfied:

$$
\lim _{h \rightarrow \theta} \frac{\left\|R\left(x_{0} ; h\right)\right\|}{\|h\|}=0 .
$$

$L\left(x_{0} ; h\right)$ is called the Fréchet differential of $F$ at $x_{0}$ and is denoted by $d F\left(x_{0} ; h\right)$. Property (2.3) can be replaced by the equivalent condition that (2.2) holds uniformly on each bounded set.

The mapping $d F\left(x_{0} ; \cdot\right)$ which is a bounded linear operator is called the Fréchet derivative of $F$ at $x_{0}$. The Gâteaux derivative $D F\left(x_{0} ; \cdot\right)$ is similarly defined.

Definition 4. If $Y=R=$ the real line and $E$ is a Hilbert space $H$, then $L\left(x_{0} ; h\right)$ is a bounded linear functional and can therefore be uniquely represented as a scalar product, i.e., there exists a unique element $\Gamma\left(x_{0}\right)$ of $H$ such that

$$
L\left(x_{0} ; h\right)=\left\langle\Gamma\left(x_{0}\right), h\right\rangle,
$$

where the notation on the right denotes the scalar product. $\Gamma\left(x_{0}\right)$ is called the gradient of $f: X \rightarrow R$ at $x=x_{0}$, and is denoted by $\operatorname{grad} f\left(x_{0}\right)$.

The variational method of proving existence and uniqueness theorems for the solutions of nonlinear operator equations is based on the construction of a functional whose critical points coincide with the solutions of the equation considered. This reformulation is possible, for instance, under the conditions expressed in the following well-known theorem [9], [15].

Theorem (Kerner's Symmetry Coxditiox). Let $F(x)$ be a mapping of a Hilbert space $H$ (or part of it) into itself. Let $F(x)$ have a Gâteaux differential $D F(x ; h)$ at every point $x$ in $S=\left\{x:\left\|x-x_{0}\right\|<r\right\}$, where $r>0$. A ssume that at every $x \in S$, the functional $\langle D F(x ; h), k\rangle$ for $h, k \in H$, is continuous in $x$. Then in order that the 
mapping $F(x)$ be the gradient of some functional $f(x)$ defined on $S$, it is necessary and sufficient that the bilinear functional $\langle D F(x ; h), k\rangle$ be symmetric for every $x \in S$, i.e.,

$$
\langle D F(x ; h), k\rangle=\langle h, D F(x ; k)\rangle \quad \text { for } h, k \in H .
$$

Under these conditions,

$$
f(x)-f\left(x_{0}\right)=\int_{0}^{1}\left\langle F\left(x_{0}+t\left(x-x_{0}\right)\right), x-x_{0}\right\rangle d t .
$$

3. General Iterative Methods Based on Variational Theory of Operators. Consider the nonlinear functional equation

$$
G(x)=\theta,
$$

where $G$ is defined on a Hilbert space $H$. For this discussion, it will be assumed that the operator $G$ satisfies Kerner's symmetry condition $\dagger$ in a sphere $S \subset H$, so that it may be identified with the gradient of a real-valued functional, say $g(x)$, defined on $H$. Assuming that sufficient conditions for the existence of a local minimum of $g(x)$ in the sphere $S$ are met, $\dagger$ some aspects of general iterative methods based on variational theory, which include the methods of gradient and steepest descents, will now be examined.

Consider the following class of iterative algorithms

$$
x_{n+1}=x_{n}+\alpha_{n} z_{n},
$$

where $\alpha_{n}$ is a sequence of real numbers and $z_{n}$ is a sequence of elements in $H$. Starting with an arbitrary element $x_{0} \in S$, choose the next approximation $x_{1}$ to the solution $x^{*}$ of (3.1) such that in the direction of $x_{0}$ to $x_{1}$, the functional $g(x)$ decreases most rapidly. This is the direction of steepest descent from $x_{0}$ to $x_{1}$.

This direction depends on the metric used to define distance. It may be recalled that if $\left[X, \rho_{1}\right],\left[X, \rho_{2}\right]$ are two metric spaces with the same set $X$, then the distance functions $\rho_{1}$ and $\rho_{2}$ are said to be topologically equivalent metrics if they define the same topology (i.e., the same open sets) on $X$. In particular, if $\|\cdot\|$ and $|\cdot|$ are two norms on the space $E$, then they are equivalent if and only if there exist positive constants $\beta$ and $\gamma$ such that

$$
\beta\|x\| \leqq|x| \leqq \gamma\|x\|
$$

for any $x \in E$. Although the definition of the Fréchet differential of a mapping $F: X \rightarrow Y$ is written in terms of the norms on $X$ and $Y$, it is easy to see that if $X$ is endowed with two equivalent norms $\|\cdot\|$ and $|\cdot|$, then $[X,\|\cdot\|]$ and $[X,|\cdot|]$ have the same Fréchet differentiable functions.

The next theorem discloses explicitly the dependence of the direction of steepest descent on the metric.

Theorem 1. Let $T_{0}$ be a self-adjoint positive-definite bounded linear operator defined on the Hilbert space $H$ with the inner product $\langle$,$\rangle ;$

$$
\left\langle T_{0} h, h\right\rangle \geqq m\langle h, h\rangle, \quad m>0, \quad \text { for all } h \in H .
$$

$\dagger$ Various relaxations of this assumption and sufficient conditions for $g(x)$ to attain a minimum in $S$ are discussed in [13] and [12], where the convergence of a generalization of the method of steepest descents is established for certain classes of nonlinear operator equations. 
For any two elements $x, y \in H$, define the distance between $x$ and $y$ by the metric

$$
\rho(x, y)=|x-y|=\left\langle T_{0}(x-y), x-y\right\rangle^{1 / 2} .
$$

Let $x_{0}$ be as before. Then the direction of steepest descent from $x_{0}$ is given by

$$
z_{0}=-T_{0}^{-1} G\left(x_{0}\right) .
$$

Proof. Let the norm of an element $x \in H$ be defined by

$$
|x|=\left\langle T_{0} x, x\right\rangle^{1 / 2} \text {. }
$$

Let $x_{1}$ be the next approximation to the solution $x^{*}$. If the metric (3.2) is used to define the distance between $x_{0}$ and $x_{1}$, then $\Gamma$, the set of all points at a distance $s$ from $x_{0}$ is given by

$$
\Gamma=\left\{x:\left\langle T_{0}\left(x-x_{0}\right), x-x_{0}\right\rangle=s^{2}\right\} .
$$

The direction of steepest descent, $h=x-x_{0}$, is determined by minimizing $g\left(x_{0}+h\right)$ subject to the constraint $x \in \Gamma$. That is,

$$
\left\langle T_{0} h, h\right\rangle=s^{2} .
$$

By the method of Lagrange's multipliers, if we set

$$
\varphi(h)=g\left(x_{0}+h\right)+\lambda\left\langle T_{0} h, h\right\rangle,
$$

then

$$
d \varphi(h ; k)=0 \quad \text { for all } k \in H .
$$

That is,

$$
\operatorname{grad} \varphi(h)=\theta .
$$

But $\operatorname{grad}\left\langle T_{0} h, h\right\rangle=2 T_{0} h$ since $T_{0}$ is self-adjoint; hence

$$
h=-\frac{1}{2 \lambda} T_{0}^{-1} G\left(x_{0}+h\right) .
$$

Eliminating $\lambda$, using (3.3) and (3.4), we get

$$
h=-s\left\langle G\left(x_{0}+h\right), T_{0}^{-1} G\left(x_{0}+h\right)\right\rangle^{-1 / 2} T_{0}^{-1} G\left(x_{0}+h\right) .
$$

Since $G\left(x_{0}\right) \neq \theta$, it follows from (3.5), using the continuity of $G(x)$ at $x=x_{0}$, that as $s \rightarrow 0$, the direction of steepest descent approaches $-T_{0}{ }^{-1} G\left(x_{0}\right)$.

Having selected $z_{0}$, the next approximation will then be

$$
x_{1}=x_{0}-\alpha_{0} T_{0}^{-1} G\left(x_{0}\right),
$$

where $\alpha_{0}$ is yet to be determined. In general, from the $n$th to the $(n+1)$ st iteration, we have

$$
x_{n+1}=x_{n}-\alpha_{n} T_{n}^{-1} G\left(x_{n}\right),
$$

where $T_{n}$ is a positive-definite self-adjoint operator defined on $H$. The parameters $\alpha_{n}$ may be chosen by any of the following procedures:

A. The Exact Optimum Gradient Method. For fixed $x_{n}, T_{n}$, the functional $g\left(x_{n+1}\right)$ 
$=g\left(x_{n}-\alpha_{n} T_{n}^{-1} G_{n}\right)$, where $G_{n}=G\left(x_{n}\right)$ is a function of $\alpha_{n}$. In the strict sense of the classical method of steepest descent, $\alpha_{n}$ should be chosen so that

$$
\eta\left(\alpha_{n}\right)=g\left(x_{n}-\alpha_{n} T_{n}{ }^{-1} G_{n}\right)
$$

is minimum. That is, $\alpha_{n}$ is taken to be the smallest positive root of the equation $d \eta(\alpha) / d \alpha=0$. This method, however, is computationally complicated as it requires the determination of $g(x)$ and a tedious nonlinear minimization problem" at each iteration.

B. The Approximate Optimum Gradient Method. In this procedure, rather than choosing $\alpha_{n}$ to minimize the exact $g\left(x_{n+1}\right)$ we minimize the second-order approximation to $g\left(x_{n+1}\right)$ obtained by using the first two terms of the Taylor series [3], [7]

$$
g\left(x_{n+1}\right) \approx g\left(x_{n}\right)-\alpha_{n}\left\langle G_{n} ; T_{n}^{-1} G_{n}\right\rangle+\frac{\alpha_{n}^{2}}{2}\left\langle D G\left(x_{n} ; T_{n}^{-1} G_{n}\right), T_{n}^{-1} G_{n}\right\rangle .
$$

Therefore

$$
\alpha_{n}=\frac{\left\langle G_{n} ; T_{n}^{-1} G_{n}\right\rangle}{\left\langle D G\left(x_{n} ; T_{n}^{-1} G_{n}\right), T_{n}^{-1} G_{n}\right\rangle} .
$$

C. Minimization of the Norm of the Residue. If the operator $G$ has a second-order Fréchet differential, an effective algorithm is obtained by choosing $\alpha_{n}$ to minimize the norm of the first-order approximation to the residue $G\left(x_{n+1}\right)$. To this end, Taylor's formula may be used to write

$$
G_{n+1}=G_{n}+D G\left(x_{n} ; x_{n+1}-x_{n}\right)+\frac{1}{2} D^{2} G\left(\bar{x}_{n} ; x_{n+1}-x_{n}, x_{n+1}-x_{n}\right),
$$

where $\bar{x}_{n}=x_{n}+\tau_{n}\left(x_{n+1}-x_{n}\right), 0<\tau_{n}<1$, and the bilinear map $D^{2} G\left(\bar{x}_{n} ; \cdot, \cdot\right)$ is the second-order Fréchet derivative of $G . \alpha_{n}$ is then chosen so that for fixed $x_{n}$

$$
\eta\left(\alpha_{n}\right)=\left\|G_{n}-\alpha_{n} D G\left(x_{n} ; T_{n}{ }^{-1} G_{n}\right)\right\|
$$

is minimum. Hence

$$
\alpha_{n}=\frac{\left\langle G_{n}, D G\left(x_{n} ; T_{n}^{-1} G_{n}\right)\right\rangle}{\left\|D G\left(x_{n} ; T_{n}^{-1} G_{n}\right)\right\|^{2}} .
$$

It is worth noting that for the case of linear equations, this reduces to the algorithms developed by Krasnosel'skir and Kreln [8] and Lanczos [10], and that the approximate optimum gradient method coincides with the exact optimum gradient method studied by Kantorovich [7].

D. Sequential Descent Methods. A larger class of algorithms of steepest descent may be obtained by determining a range for $\alpha_{n}$ within which the sequence (3.6) converges to a solution of (3.1). This approach is more useful than the other descent methods since it allows a choice of $\alpha_{n}$ and $T_{n}$ which leads to a rapid rate of convergence of the iteration process [12].

In any of the above procedures, a sequence $x_{0}, x_{1}, \cdots, x_{n}, \cdots$ is generated. Let $P$ be the broken-line path of steepest descent. The path $P$ may end at a point $x^{*}$ where the direction of steepest descent does not exist, i.e., $G\left(x^{*}\right)=\theta$, or may continue indefinitely lying entirely within the sphere $S$, or may cross the boundary of $S$. It is shown in [13] that under certain conditions on $\alpha_{n}, T_{n}$ and $G$, the last 
possibility can be avoided. In the subsequent discussion, it will be assumed that the path $P$ continues indefinitely within $S$.

Theorem 2. Let $G(x)$ satisfy Kerner's symmetry condition. Let $G\left(x_{0}\right) \neq \theta$ and let there exist a convex neighborhood $\Omega$ of $x_{0}$ such that the linear operator $d G(x ; \cdot)$ is continuous in $x$ on $\Omega$. If $x_{n}, x_{n+1} \in \Omega$, then for $\alpha_{n}$ sufficiently small and positive

$$
g\left(x_{n+1}\right)<g\left(x_{n}\right), \quad n=0,1,2, \cdots .
$$

Proof. The scalar product $\langle G(x), k\rangle$ has for each $k$ a Fréchet differential at $x$ which is equal to $\langle d G(x ; h), k\rangle$. This follows from the definition of the Fréchet differential. In fact

$$
\langle G(x+h)-G(x), k\rangle=\langle d G(x ; h), k\rangle+\langle R(x ; h), k\rangle,
$$

where

$$
\lim _{\|h\| \rightarrow 0} \frac{\|R(x ; h)\|}{\|h\|}=0
$$

Therefore,

$$
\lim _{\|h\| \rightarrow 0}\|h\|^{-1}\{\langle G(x+h)-\dot{G}(x), k\rangle-\langle d G(x ; h), k\rangle\}=\lim _{\|h\| \rightarrow 0} \frac{\langle R(x ; h), k\rangle}{\|h\|}=0 .
$$

Hence, $g(x)$ has a second-order differential $d^{2} g(x ; h, k)=\langle d G(x ; h), k\rangle$ for $x \in \Omega$. By continuity of $d G(x ; h), d^{2} g(x ; h, k)$ is continuous in $x$, for $x \in \Omega$ and is symmetric in $h$ and $k$ (Theorem 8 in [3] or Satz 1 in [9]). Therefore, Taylor's formula [3, Theorem 5] may be used to get

$$
g\left(x_{n+1}\right)-g\left(x_{n}\right)=-\alpha_{n}\left\langle G_{n}, T_{n}^{-1} G_{n}\right\rangle+R_{2},
$$

where

$$
\begin{aligned}
R_{2} & =\int_{0}^{1}(1-t) d^{2} g\left(x_{n}+t\left(x_{n+1}-x_{n}\right) ; x_{n+1}-x_{n}, x_{n+1}-x_{n}\right) d t \\
& =\alpha_{n}{ }^{2} \int_{0}^{1}(1-t)\left\langle d G\left(x_{t} ; T_{n}^{-1} G_{n}\right), T_{n}^{-1} G_{n}\right\rangle d t
\end{aligned}
$$

and

$$
x_{t}=x_{n}+t\left(x_{n+1}-x_{n}\right), \quad 0<t<1 .
$$

The theorem then follows using (3.7) and (3.8) and noting that $\alpha_{n}$ and $\left\langle G_{n}, T_{n}{ }^{-1} G_{n}\right\rangle$ are positive.

4. Iterative Methods Based on the Relative Error Functional. Consider the functional equation

$$
G(x)=\theta
$$

and assume that it has a solution $x^{*}$ in a certain bounded convex set $\Omega$ of a Hilbert space $H$. If $x_{n}$ is a sequence of approximations to the exact solution $x^{*}$ generated by a certain algorithm, we define for the $n$th iterate, the error $s_{n}$ and the residue $r_{n}$ respectively by

$$
s_{n}=x_{n}-x^{*}
$$


and

$$
r_{n}=G\left(x_{n}\right) .
$$

For $x \in \Omega$, define the relative error functional by

$$
e(x)=\langle s, Q r\rangle=\left\langle x-x^{*}, Q G(x)\right\rangle,
$$

where $Q$ is a certain bounded linear operator. Clearly, $e(x)$ gives some measure of the derivation of the approximation $x_{n}$ from the exact solution $x^{*}$.

Let $G$ be Gâteaux differentiable on $\Omega$, then

$$
\begin{aligned}
D e(x ; h) & =\lim _{t \rightarrow 0} \frac{e(x+t h)-e(x)}{t} \\
& =\lim _{t \rightarrow 0}\left\{\left\langle x-x^{*}, Q\left(\frac{G(x+t h)-G(x)}{t}\right)\right\rangle+\langle h, Q G(x+t h)\rangle\right\} \\
& =\left\langle x-x^{*}, Q D G(x ; h)\right\rangle+\langle h, Q G(x)\rangle \\
& =\left\langle[Q D G(x ; \cdot)]^{*}\left(x-x^{*}\right)+Q G(x), h\right\rangle,
\end{aligned}
$$

where the asterisk denotes the adjoint of the bounded linear operator $Q D G(x ; \cdot)$. Hence

$$
\operatorname{grad} e(x)=Q G(x)+[Q D G(x ; \cdot)]^{*}\left(x-x^{*}\right) .
$$

The next theorem shows that the problem of solving (4.1) is closely related to the functional $e(x)$.

Theorem 3. Let $G$ be Gateaux differentiable on the convex set $\Omega$ and assume that for any $x, x+h \in \Omega$ and real $t$,

$$
\left.\left\langle\frac{d}{d t} Q G(x+t h), h\right\rangle\right|_{t=0} \geqq m\|h\|^{2},
$$

where $m>0$. Then $e(x)=0$ and $\operatorname{grad} e(x)=\theta$ if and only if $x=x^{*}$, where $x^{*}$ is the solution of (4.1) in $\Omega$.

Proof. The sufficiency is evident. To prove the necessity, suppose there exists an element $u \in \Omega, u \neq x^{*}$ such that $e(u)=0$ and $\operatorname{grad} e(u)=\theta$. Then, considering the real-valued function $\left\langle x^{*}-u, Q G\left(t u+(1-t) x^{*}\right)\right\rangle$ for $0 \leqq t \leqq 1$ and applying Rolle's theorem we get

$$
\left.\frac{d}{d t}\left\langle x^{*}-u, Q G\left(t u+(1-t) x^{*}\right)\right\rangle\right|_{t=\xi}=0,
$$

where $0<\xi<1$. That is, letting $x=\xi u+(1-\xi) x^{*}$, we get

$$
\begin{aligned}
0=\lim _{\Delta t \rightarrow 0}(\Delta t)^{-1}\left\langle u-x^{*}, Q G\right. & \left.\left(x+\Delta t\left(u-x^{*}\right)\right)-Q G(x)\right\rangle \\
& =\lim _{\tau \rightarrow 0} \tau^{-1}(1-\xi)^{-2}\langle u-x, Q G(x+\tau h)-Q G(x)\rangle,
\end{aligned}
$$

where

$$
\tau=\frac{\Delta t}{1-\xi}, \quad h=u-x
$$


Hence,

$$
\left\langle u-x,\left.\frac{d}{d \tau} Q G(x+\tau h)\right|_{\tau=0}\right\rangle=0 .
$$

On the other hand, since $x, x+h \in \Omega$, we have by assumption

$$
\left\langle u-x,\left.\frac{d}{d \tau} Q G(x+\tau h)\right|_{\tau=0}\right\rangle \geqq m\|u-x\|^{2}, \quad m>0 .
$$

Consequently $x=x^{*}=u$.

If $\operatorname{grad} e(u)=\theta$, then by $(4.2)$,

$$
Q G(u)-Q G\left(x^{*}\right)=[Q D G(u ; \cdot)]^{*}\left(x^{*}-u\right) .
$$

Taking the inner product of the last equation with $\left(x^{*}-u\right)$ and using a mean value theorem argument as in the first part of this theorem we arrive at a contradiction unless $u=x^{*}$.

For linear operators the following stronger theorem holds:

Theorem 4. Let

$$
G(x)=A x-b=\theta
$$

where $A$ is a bounded linear operator defined on $H$ and $b$ is a fixed element. Let $Q$ be an invertible operator defined on $H$ and assume that $A$ is $Q$-symmetric and $Q$-positive definite, i.e.,

$$
\langle Q A x, x\rangle \geqq C\|Q x\|^{2}, \quad x \in H, \quad C>0 .
$$

Given any linear subspace $L$ of $H$ and any element $x \in H$, then

$$
e(x) \leqq e(x+h)
$$

for all $h \in L$ if and only if $\operatorname{grad} e(x)$ is orthogonal to $L$. In particular $x$ minimizes $e(x)$ if and only if $x=x^{*}$, where $x^{*}$ is the solution of (4.3).

Proof. Let $h$ be any nonnull element of $L$ and let $x$ be an arbitrary element in $H$. Then

$$
\begin{aligned}
e(x+h)-e(x) & =\left\langle Q G(x+h), x-x^{*}+h\right\rangle-\left\langle Q G(x), x-x^{*}\right\rangle \\
& =\langle Q G(x), h\rangle+\langle Q A h, h\rangle+\left\langle Q A h, x-x^{*}\right\rangle \\
& =2\langle Q G(x), h\rangle+\langle Q A h, h\rangle .
\end{aligned}
$$

Also, since $d G(x ; h)=A h$ in this case, we get

$$
\operatorname{grad} e(x)=Q G(x)+Q A x-Q A x^{*}=2 Q G(x) .
$$

Thus we have

$$
e(x+h)-e(x)=\langle\operatorname{grad} e(x), h\rangle+\langle Q A h, h\rangle .
$$

Therefore if $\operatorname{grad} e(x)$ is orthogonal to the subspace $L$, the first term on the righthand side of (4.4) vanishes, and in view of the assumption made on $Q A$, it follows that

$$
e(x+h)-e(x) \geqq 0 .
$$


This disposes of the sufficiency. To prove the necessity, assume that there exists an $x \in H$ such that for all $h \in L, e(x) \leqq e(x+h)$. Since $L$, by assumption, is a subspace of $H$, we have for any real number $t$, and an arbitrary nonnull element $h \in L$,

$$
\begin{aligned}
0 \leqq e(x+t h)-e(x)= & t^{2}\langle Q A h, h\rangle+t\langle\operatorname{grad} e(x), h\rangle \\
= & \langle Q A h, h\rangle\left[t-\frac{\langle\operatorname{grad} e(x), h\rangle}{2\langle Q A h, h\rangle}\right]^{2} \\
& -\frac{\langle\operatorname{grad} e(x), h\rangle^{2}}{4\langle Q A h, h\rangle} .
\end{aligned}
$$

Choosing $t$ such that the term in the bracket vanishes, we arrive at the conclusion that $\langle\operatorname{grad} e(x), h\rangle=0$.

Remark. This theorem is a generalization of a result stated by Antosiewicz and Rheinboldt [16, Theorem 14.2]. The proof given there, however, contains a misprint.

, The variational approach to the solution of equation (4.3) would involve the functional

$$
g(x)=\frac{1}{2}\{\langle Q A x, x\rangle-\langle Q x, b\rangle-\langle Q b, x\rangle\} .
$$

Indeed, it may be easily verified that $Q^{-1} \operatorname{grad} g(x)=G(x)=A x-b$ and that a necessary and sufficient condition for $x^{*}$ to be the solution of $(4.3)$ is that $x^{*}$ minimize $g(x)$.

For the class of linear operator equations defined in Theorem 4, we have, on the other hand, using the relative error functional,

$$
e(x)=\langle Q A x, x\rangle-\langle Q x, b\rangle-\langle Q b, x\rangle+\left\langle Q b, x^{*}\right\rangle .
$$

Thus the following relation is obtained:

$$
e(x)=2 g(x)+\left\langle Q b, x^{*}\right\rangle .
$$

This relation shows that the iterative methods based on the minimization of the relative error functional and the gradient methods are the same for linear operator equations. In both cases, we seek to minimize the same measure of the error. It may be noted that while the relative error functional is defined in terms of the error $s$, which is unavailable for computational purposes, relation (4.6) shows that this difficulty is only apparent in the case of linear operator equations. Crucial to this simplification is the fact that the residue and the error are related by the equation $r=A s$.

Returning to the iterative methods based on the consideration of the relative error function, for nonlinear functional equations, we seek, in view of Theorem 3, to minimize either the functional $e(x)$ or $\|\operatorname{grad} e(x)\|$. The only difficulty, however, is that these quantities are defined in terms of the error $s$, which cannot be eliminated. To overcome this difficulty, we replace $s$ by the first-order approximation $\left[Q D G\left(x_{n} ; \cdot\right)\right]^{-1} Q G\left(x_{n}\right)$. It is evident that this gives the exact value of $s$ for the linear operators of Theorem 3. 
We may then proceed to minimize $e(x)$ successively in the directions $p_{1}, p_{2}$, $\cdots, p_{n}, \cdots$ of linearly independent vectors determined by some algorithm. This gives

$$
\begin{aligned}
x_{n+1} & =x_{n}-\alpha_{n} p_{n}, \quad n=0,1,2, \cdots, \\
e\left(x_{n+1}\right) & =\left\langle x_{n}-x^{*}-\alpha_{n} p_{n}, Q G\left(x_{n}-\alpha_{n} p_{n}\right)\right\rangle \\
& \approx\left\langle\left[Q D G\left(x_{n} ; \cdot\right)\right]^{-1} Q G\left(x_{n}\right)-\alpha_{n} p_{n}, Q G\left(x_{n}\right)-\alpha_{n} Q D G\left(x_{n} ; p_{n}\right)\right\rangle
\end{aligned}
$$

so that the minimum of $e\left(x_{n+1}\right)$ in the direction $p_{n}$ is approximately obtained for

$$
\alpha_{n}=\frac{\left\langle p_{n}, Q G\left(x_{n}\right)\right\rangle}{\left\langle p_{n}, Q D G\left(x_{n} ; p_{n}\right)\right\rangle} .
$$

Various choices of $p_{n}$ and $Q$ lead to algorithms mentioned in the introduction of this paper for the case of linear operator equations.

5. Iterative Algorithms Based on the Projection Method. The class of projection methods considered by Gastinel [2], and Householder and Bauer [6] for solving linear algebraic systems of equations and by Petryshyn [14] for linear operator equations involves the resolution of $s_{n}$ into two components. One of these is $s_{n+1}$, and is required to be smaller than $s_{n}$ in some norm; the other belongs to some subspace selected at each iteration. In considering the projection method for solving nonlinear functional equations, an invertible operator $Q$ may be selected and the error $s_{n+1}$ is resolved in the form

$$
s_{n+1}=s_{n}-\alpha_{n} Q r_{n} .
$$

$\alpha_{n}$ is to be chosen so that the element $\alpha_{n} Q r_{n}$ is the orthogonal projection of the vector $s_{n+1}$ on $Q r_{n}$ in the sense of the metric induced by the scalar product $[x, y]=$ $\left\langle T_{n} x, y\right\rangle$, where $T_{n}$ is a positive definite self-adjoint linear operator. Hence

$$
\alpha_{n}=\frac{\left\langle T_{n} s_{n}, Q r_{n}\right\rangle}{\left\langle T_{n} Q r_{n}, Q r_{n}\right\rangle} .
$$

This value of $\alpha_{n}$ minimizes the norm of the error vector $s_{n+1}$. The numerical computations cannot be carried out until the operators $T_{n}$ and $Q$ are specified. Moreover, these must be specified in such a way that $s_{n}$ is eliminated from (5.1). In considering nonsingular linear operator equations, one choice which suggests itself is $T_{n}=B_{n} A$; then $T_{n} s_{n}=B_{n} r_{n}$. Suitable choices for $B_{n}$ would be $A^{*}$ or $A$.

For nonlinear operator equations, we shall again replace $s_{n}$ in (5.1) by the firstorder approximation $\left[Q D G\left(x_{n} ; \cdot\right)\right]^{-1} Q G\left(x_{n}\right)$. Several specializations of $\alpha_{n}$ lead to the methods based on the variational theory discussed in Section 3.

Georgia Institute of Technology

Atlanta, Georgia

1. M. Altman, "A general method of steepest ortho-descent," Bull. Acad. Polon. Sci. Ser. Sci. Math. Astronom. Phys., v. 9, 1961, p. 645-651. MR 25 \#526.

2. N. Gastinel, "Procédé itératif pour la résolution numérique d'un système d'équations linéaires," C. R. Acad. Sci. Paris, v. 246, 1958, p. 2571-2574. MR 20 * 1404.

3. L. M. GRAvEs, "Riemann integration and Taylor's theorem in general analysis," Trans. Amer. Math. Soc., v. 29, 1927, p. 163-177. 
4. R. M. HAYES, "Iterative methods of solving linear problems on Hilbert space," Contributions to the Solution of Systems of Linear Equations and the Determination of Eigenvalues, Nat. Bur. Standards Appl. Math. Ser., No. 39, U. S. Government Printing Office, Washington, D. C., 1954, p. 71-103. MR 16, 597.

5. M. R. Hestenes, "Hilbert space methods in variation theory and numerical analysis," Proc. Internat. Congress of Mathematicians, v. 3, 1954, p. 229-236.

6. A. S. HOUSEHOLDER \& F. L. BAUER, "On certain iterative methods for solving linear systems," Numer. Math., v. 2, 1960, p. 55-59. MR 22 *7251.

7. L. V. KaNTOROVICH \& G. P. AkILOv, Functional Analysis in Normed Spaces, Fizmatgiz, Moscow, 1959. (Russian) MR $22 * 9837$.

8. M. A. KRASNOSEL'SKIĬ \& S. G. KREǏN, "An iteration process with minimal residuals," Mat.Sb. (N.S.), v. 31 (73), 1952, p. 315-334. MR 14, 692.

9. M. Kerner, "Die Differentiale in der allgemeinen Analysis," Ann. of Math., v. 34, 1933, p. 546-572.

10. C. LANCZOS, "Solution of systems of linear equations by minimized iterations," J. Res. Nat. Bur. Standards, v. 49, 1952, p. 33-53. MR 14, 501.

11. Ju. Lumiste, "The method of steepest descent for nonlinear equations," Tartu. Gos. Univ. Trudy Estest.-Mat. Fak., v. 37, 1955, p. 106-113. (Russian. Estonian Summary) MR 17, 900 .

12. M. Z. NASHED, "Iterative methods for the solutions of nonlinear operator equations in Hilbert space," Ph. D. Dissertation, The University of Michigan, Ann Arbor, Mich., 1963.

13. M. Z. NASHED, "The convergence of the method of steepest descents for nonlinear equations with variational or quasi-variational operators," J. Math. Mech., v. 13, 1964, p. 765-794.

14. W. V. Petryshyn, "Direct and iterative methods for the solution of linear operator equations in Hilbert space," Trans. Amer. Math. Soc., v. 105, 1962, p. 136-175. MR 26 *3180.

15. E. H. Rothe, "Gradient mappings," Bull. Amer. Math. Soc., v. 59, 1953, p. 5-19. MR $14,657$.

16. J. Todd, Ed., Survey of Numerical Analysis, McGraw-Hill, New York, 1962.

17. M. M. VAǏnBerg, Variational Methods for Investigation of Non-Linear Operators, GITTL, Moscow, 1956. (Russian) MR 19, 567.

18. M. M. VAǏNBERG, "On the convergence of the method of steepest descents for nonlinear equations," Dokl. Akad. Nauk SSSR, v. 130, 1960, p. 9-12. Soviet Math Dokl., v. 1, 1960, p.1-4. MR $25 * 751$.

19. M. M. VAǏNBERG, "On the convergence of the process of steepest descent for nonlinear equations," Sibirsk. Mat. Ż., v. 2, 1961, p. 201-220. (Russian) MR 23 *A4026. 